\title{
Ethical Issues to be Considered for the Neurosurgeons During the COVID-19 Pandemic
}

Mohammed Maan Al-Salihi ${ }^{1}$, Maryam Sabah Al-Jebur1 ${ }^{1}$ Sabrina Rahman ${ }^{2}$ Ivan David Lozada-Martinez ${ }^{3}$, Md Moshiur Rahman,*

${ }^{1}$ College of Medicine/ University of Baghdad, Baghdad, Iraq

${ }^{2}$ Department of Public health, Independent University-Bangladesh, Dhaka, Bangladesh

${ }^{3}$ Medical and Surgical Research Center, School of Medicine, University of Cartagena, Cartagena, Colombia

${ }^{4}$ Neurosurgery Department, Holy Family Red Crescent Medical College, Dhaka, Bangladesh

*Corresponding author: Md Moshiur Rahman, Department of Neurosurgery, Holy Family Red Crescent Medical College, Dhaka, Bangladesh

Received date: 1 November, 2021

Accepted date: 9 November, 2021|

Published date: 12 November, 2021

Citation: Al-Salihi MM, Al-Jebur MS, Rahman S, Martinez IDL, Rahman MM. (2021) Ethical Issues to be Considered for the Neurosurgeons During the COVID-19 Pandemic. J Anaesth Anesth Drug 1(1). doi https://doi.org/10.54289/JAAD2100104

Copyright: (c) 2021 Al-Salihi MM, et al. This is an open-access article distributed under the terms of the Creative Commons Attribution License, which permits unrestricted use, distribution, and reproduction in any medium, provided the original author and source are credited. Abbreviations: PPE: personal protective equipment, TBI: traumatic brain injury.

\section{Dear editor,}

We read many articles about the neurosurgeons' experience during the covid-19 pandemic with a great interest, and we were interested in their handling of a topic of a rising importance for neurosurgeons during the Coronavirus Disease 2019 (COVID-19) pandemic. Reading Finn et al [1] discussion about the medico-legal considerations about neurosurgeons' practice in response to COVID-19, and the challenges experienced with using personal protective equipment (PPE) encouraged us to dig deeper in the ethical consideration for neurosurgical practice during COVID-19 pandemic.

All physicians learn during their early years of medical schools that their safety is a priority even during lifesaving emergencies [2]. Ensuring their safety is, for example, the first step in cardiopulmonary resuscitation [3]. During the COVID-19 pandemic, a significant debate was raised concerning the ethical consideration of physicians' safety during such a life-threating pandemic4. Several authors have handled the topic from different perspectives [4-6].

The balance between physicians' safety, patients' optimized care, urgency of patients' care needed, and limited resources were agreed factors that should be considered during ethical recommendations [4-6].

Adding to what we have read concerning ethical issues for the neurosurgeons during the COVID-19 pandemic, the main issue we want to shed the light on is the relative urgency of neurosurgical procedures in comparison to other surgeries. This is essential for patients' optimized care (or duty to care). It is estimated that at least 75 - 115 individuals per 100000 population need urgent neurosurgical interventions annually [7], and delay in such inventions might result in significant morbidity and/or mortality [8]. During COVID-19 pandemic, there was a decline in approximately one third (33.6\%) of the rates of emergent neurosurgical referrals and more than half $(55.6 \%)$ of the emergent intervention [9].

As duty to care has no less weight than the physician safety, it is worth mentioning that patients needing neurosurgical interventions are special group of patients whose diseases are time sensitive. As a role in neurosurgery, time is brain [10]. Unlike most of the body cells and tissues, neurons exposed to insults (e.g. compression, ischemia, epileptic activity, trauma, etc.) are prone to irreversible damage as the time passes [11]. This puts a challenging ethical burden to postponing most of 
neurosurgical interventions, even elective ones, for safety issues 11 . The longer the time the patient waits prior to his surgery, the less likelihood he might restore his function.

Whilst the rates of emergent neurosurgical interventions declined significantly during the pandemic, the results reported by Grassner et al. Whilst the rates of emergent neurosurgical interventions declined significantly during the pandemic, the results reported by Grassner et al TBI), which constitutes a significant proportion (more than $80 \%$ ) of neurosurgical interventions $[\mathbf{1 2 , 1 3}]$. The vast majority of neurosurgical interventions are indicated for traumatic brain injury (TBI), stroke-related conditions, tumors, hydrocephalus and epilepsy [14].

The non-significant 30-day mortality reported by Grassner et al. [12] despite the declining rates of non-urgent neurosurgical interventions was assuring for many patients and physician. However, the authors did not evaluate patients' morbidity and quality of life [12]. The 'stay home' recommendations and legal regulations to reduce crowd during the COVID-19 pandemic which reduced the overall incidence of TBI, and subsequently urgent neurosurgical interventions [13]. The non-significant 30-day mortality reported by Grassner et $a$. [14] despite the declining rates of non-urgent neurosurgical interventions was assuring for many patients and physician. However, the authors did not evaluate patients' morbidity and quality of life. [15] both mortality and long-term morbidity are urgently needed. Ethical considerations should include a band for those patients whose quality of life and disability are at high risk. Postponing neurosurgical intervention in such cases victimizes their long-term outcome [16]. A nice, proposed approach to select and prioritize non-elective patients is to use functionality rather than electivity to triage neurosurgical interventions during the COVID-19 pandemic [17]. Expert neurosurgeons are needed to develop consensus recommendations for management of both elective and nonelective cases based on their functionality, expected prognosis, window of opportunity available for appropriate management before rescuing their nervous tissue, comorbidities, and available human and non-human resources. Implementation of such an approach would save patients with ischemic cerebrovascular stroke coming in window of thrombectomy from long-term disability, save patients with refractory epilepsy from the deleterious complications of seizures and status epilepticus, prevent acute complications of hydrocephalus and optimize the outcome of non-traumatic hemorrhage such as ruptured aneurysms or vascular malformations.

\section{Conflict of Interest: None.}

\section{Disclosure of Funding: None.}

\section{Reference}

1. Abdelgadir J, Smith ER, Punchak M, et al. (2017) Epidemiology and Characteristics of Neurosurgical Conditions at Mbarara Regional Referral Hospital. World Neurosurg. 102: 526-532.

2. Ashkan K, Jung J, Velicu AM, et al. (2021) Neurosurgery and coronavirus: impact and challengeslessons learnt from the first wave of a global pandemic. Acta Neurochir (Wien). 163(2): 317-329.

3. Bakewell F, Pauls MA, Migneault D. (2020) Ethical considerations of the duty to care and physician safety in the COVID-19 pandemic. Can J Emerg Med. 22(4): $407-$ 410.

4. Finn R, Ganau M, Jenkinson MD, Plaha P. (2021) COVID-legal study: neurosurgeon experience in Britain during the first phase of the COVID-19 pandemicmedico-legal considerations. Br J Neurosurg. Published online. 24: 1-4.

5. González MLG, Alegre MS, Roda JM. (2020) Neurosurgeons on the frontline of COVID-19: no place for surgery? Acta Neurochir (Wien). 162(7): 1503-1504.

6. Grassner L, Petr O, Warner FM, et al. (2021) Trends and outcomes for non-elective neurosurgical procedures in Central Europe during the COVID-19 pandemic. Sci Rep. 11(1): 6171.

7. Kramer JB, Brown DE, Kopar PK. (2020) Ethics in the Time of Coronavirus: Recommendations in the COVID19 Pandemic. J Am Coll Surg. 230(6): 1114-1118.

8. Kwon DH, Kim KT. (2020) Keep "time is brain," even in the crisis of COVID-19. Neurol Sci. 41(7): 1655-1656. 
9. Understanding delays in acute stroke care: A systematic review of reviews. Eur J Public Health. 28(3): 426-433.

10. Merritt Hawkins. (2018) Physician Supply Considerations: The Emerging Shortage of Medical Specialists.

11. Persad GC. (2020) A Conceptual Framework for Clearer Ethical Discussions About COVID-19 Response. Am J Bioeth. 20(7): 98-101.

12. Saver JL. (2006) Time is brain - Quantified. Stroke. 37(1): 263-266.

13. Schuhmann MU, Rickels E, Rosahl SK, Schneekloth CG, Samii M. (2001) Acute care in neurosurgery: Quantity, quality, and challenges. J Neurol Neurosurg Psychiatry. 71(2): 182-187.

14. Shlobin NA, Rosenow JM, Ford PJ. (2020) Using Functionality Rather than Elective Nature to
Characterize Neurosurgeries During Pandemic Triage. Am J Bioeth. 20(7): 196-198.

15. Sulmasy LS, Bledsoe TA. (2019) American College of Physicians Ethics Manual: Seventh Edition. Ann Intern Med. 170(2): S1-S32.

16. Tam CW, Kumar SR, Ivascu NS. (2018) Cardiopulmonary resuscitation. In: Pharmacology and Physiology for Anesthesia: Foundations and Clinical Application 575-584.

17. Vaca SD, Kuo BJ, Vissoci JRN, et al. (2019) Temporal delays along the neurosurgical care continuum for traumatic brain injury patients at a Tertiary Care Hospital in Kampala, Uganda. Clin Neurosurg. 84(1): 95-103. 